\title{
As quedas no cenário da velhice: conceitos básicos e atualidades da pesquisa em saúde
}

\author{
Falls in elderly: basics concepts and updates of research in health
}

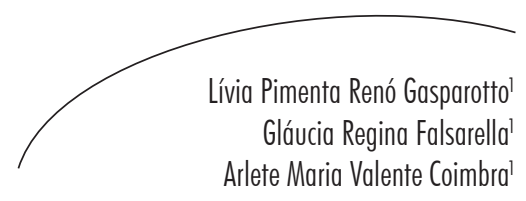

\section{Resumo}

Quedas têm sido referidas como importante fator de risco para redução da capacidade funcional em indivíduos idosos. Por sua alta prevalência nesta população, um dos objetivos das pesquisas foi delinear a queda sob seus aspectos causais e respectivas consequências. Com base nessas definições, é possível apontar perspectivas para a atuação da saúde na mitigação dos efeitos deste evento, bem como reduzir o alto gasto hospitalar destes idosos para a saúde pública. Dentro da lógica citada, este trabalho traz algumas das principais considerações e consensos da atualidade sobre queda, a partir da análise de pesquisas recentes. Tem como objetivo propor uma visão ampliada, capaz de divulgar os aspectos ligados à queda que são de interesse comum a todos os profissionais de saúde que lidam com essa população. Metodologia: utilizaram-se as bases Cochrane, Pubmed e Medline para busca das informações pretendidas através de descritores correspondentes às palavras Envelhecimento e Quedas e tendo como limite os anos 2009 a 2013. Resultados: Definiu-se para esta discussão os artigos ligados a aspectos epidemiológicos, fatores associados, consequências da queda, prevenção e intervenção nas quedas. Conclusão: Em resumo, o suporte multiprofissional está alicerçado nas práticas preventivas de quedas e necessita, portanto, de uma postura compartilhada de informações entre os profissionais da saúde que trabalham com este público.

\section{Abstract}

Falls have been reported as an important risk factor for reduced functional capacity in elderly subjects. Because of its high prevalence in this population, one of the goals of the research is to delineate the aspects falling under their respective causes and consequences. From these definitions it is possible to point out perspectives for health action in mitigating the effects of this event as well as reduce the high cost of services in the hospital to public health. Within this logic, this work presents some key considerations and consensus of fall, from the analysis of recent research. It aims to propose a broader view, able to disclose aspects of fall that is of common interest to all health professionals who works with this population group. Methods: we used

Palavras-chave: Acidentes por Quedas. Idoso. Envelhecimento.
Key words: Accidental Falls. Elderly. Aging.

Faculdade de Ciências Médicas. Universidade Estadual de Campinas. Campinas, SP, Brasil. 
the Cochrane, Medline and Pubmed databases to search the information sought by the corresponding descriptors Ageing and Falls and having as limit the years 2009 to 2013. Results: We defined for this discussion articles related to epidemiology, associated factors, consequences, fall prevention and intervention in falls. Conclusion: In summary, the multidisciplinary support is based in the preventive practices of falls and therefore needs an attitude of shared information between health professionals who work with this people.

\section{INTRODUÇÃOO}

O aumento da população de idosos, já notado em nações desenvolvidas, direciona-se agora para países como o Brasil. ${ }^{1}$ Com isso, seja no âmbito de pesquisas ou de cuidados, ambos direcionam a atenção para propostas que visem à manutenção da saúde nessa população. ${ }^{2}$ Este viés parte do pressuposto de que, ao atingir a terceira idade, os idosos apropriam-se ao longo dos anos de afecções características do quadro das doenças crônico-degenerativas. Assim, ao profissional de saúde cabe não somente a tarefa de propor, mas de sensibilizar o paciente sobre a importância de buscar no andamento da enfermidade as maneiras de se obter o controle e a manutenção da funcionalidade. ${ }^{3,4}$

Ao pensar em funcionalidade para pessoas idosas, um termo muito utilizado no campo da Gerontologia é a capacidade funcional. ${ }^{5} \mathrm{Tal}$ característica é divulgada como uma ferramenta que compõe um dos parâmetros da qualidade de vida nesta população e é expressa por meio da independência funcional (habilidade para executar tarefas do cotidiano) e autonomia funcional (capacidade de decidir, delegar funções) sem que nas duas opções seja tão necessário o pedido de ajuda.,

O processo de envelhecimento naturalmente promove modificações no corpo. No caso do idoso, é comum identificar parâmetros reduzidos da massa muscular que reduzem força, assim como os de densidade óssea, que enfraquecem o componente esquelético do indivíduo, fragilizando-0. ${ }^{7,8}$ Estes aspectos refletem na sua postura, na maneira de andar, no equilíbrio, fatores que podem facilitam o evento da queda. ${ }^{9,10}$
A queda é definida como um evento não intencional que tem como resultado a mudança da posição inicial do indivíduo para um mesmo nível ou nível mais baixo. No entanto, não há definição padronizada em função de alguns estudiosos ainda discutirem, por exemplo, se a queda abrange também alguns tipos de tropeços e escorregões. ${ }^{11,12}$

A queda, apesar de ser um fator externo ao indivíduo, decorre, na velhice, da junção de todos os fatores já citados que contemplam a chamada "incapacidade funcional". Apresenta importância relevante no cenário do envelhecimento populacional por acometer número representativo neste grupo., ${ }^{2,3}$ Sua recorrência (que também é comum) gera déficits que impactam fisicamente no processo de fragilização e compromete aspectos psicossociais do indivíduo idoso. É um tema que merece o conhecimento não só daqueles que são vítimas deste processo, mas de todos os que apresentam relação direta com pessoas idosas, principalmente os profissionais de saúde. ${ }^{8,12}$

O artigo tem como objetivo propor uma visão ampliada, capaz de divulgar os aspectos ligados à queda que são de interesse comum a todos os profissionais de saúde que lidam com o envelhecimento humano.

\section{METODOLOGIA}

A fim de atingir o objetivo proposto, de rever o tema "queda" sob a perspectiva de atualização, a pesquisa priorizou estudos recentes encontrados em bases referenciais de pesquisa (PubMed, Cochrane e Medline). Para tanto, optou-se 
por recrutar artigos de abrangência nacional e internacional, determinando-se descritores nas línguas inglesa e portuguesa. As buscas foram selecionadas entre maio e junho de 2013. As palavras escolhidas foram aquelas relacionadas, primeiramente, ao envelhecimento (elderly, aging, olderpeople, idosos, envelhecimento) e, em seguida, quedas (falls, quedas).

Como critérios de inclusão, foram priorizados os estudos mais atualizados (entre 2009 e 2013). Apesar deste pré-requisito, foram também incluídas referências de base importante para a discussão de quedas, mesmo não apresentando publicação recente. A seleção preocupou-se em absorver artigos de revisão e/ou metanálise.

Para melhor adequação do tema "queda" ao que se pretende informar no presente estudo, os resultados da busca foram separados em subtítulos que norteiam e conceituam os delineamentos de pesquisa em quedas. São eles: aspectos epidemiológicos, fatores associados, consequências da queda, prevenção e intervenção nas quedas.

\section{RESULTADOS E DISCUSSÃO}

A dimensão das quedas na população mundial e nacional traz importantes aplicações no campo da saúde. Para entender a complexidade com que o tema se relaciona ao público de idosos, o artigo traz os principais aspectos discutidos pela comunidade científica, e que servem de base na aplicabilidade de intervenções preventivas nas áreas da saúde.

\section{Aspectos epidemiológicos}

Dados apontam que 28 a $35 \%$ de pessoas acima de 65 anos de idade caem pelo menos uma vez durante o ano no mundo. A proporção aumenta para até $42 \%$ quando os idosos têm mais de 70 anos. ${ }^{1,13}$

Estudos epidemiológicos com idosos brasileiros mostram que as quedas atingem de
30 a 40\%. Uma amostra verificou que 10,8\% dos idosos relataram duas ou mais quedas com prevalência de fratura óssea de 5,2\%. Outra análise apontou que $12,1 \%$ tiveram fratura como consequência. Com isso, nota-se que a frequência dessas quedas sofre influência da progressão da idade e do nível de fragilidade, fazendo com que a cada ano, no Brasil, cerca de 10\% da população com idade acima de 75 anos perca a independência em uma ou mais atividades da vida diária. ${ }^{5,8,13,14}$

Ainda se tratando do número de idosos por idade que caem no Brasil, os estudos apontavam, há quase uma década, que a proporção desta ocorrência ficava em torno de $32 \%$ entre 65 e 74 anos, 35\% entre 75 e 84 anos e 51\% após essa idade. Isso confirma que o nível de fragilidade, entre outros, aumenta com o passar dos anos e possibilita em maior proporção os acidentes. ${ }^{15,16}$

Estudos estimam que 60 a $70 \%$ das quedas em idosos ocorrem dentro de seus lares e esta proporção pode aumentar com o passar da idade, observada em indivíduos acima de 75 anos. Dentre as quedas que geram fratura de fêmur, um estudo verificou que $30 \%$ deste público morre em até um ano. ${ }^{17-20}$

A queda, entre os diagnósticos de admissão em hospitais, é um dos motivos que mantêm por mais tempo o indivíduo internado, fato este que gera mais gasto para a saúde pública. Entre alguns países do norte da América e Europa, verificou-se que o tempo de permanência no hospital costuma variar de quatro a 15 dias. Isso se prolonga ainda mais em países em desenvolvimento ou subdesenvolvidos cujas políticas de saúde sofrem com falta de suporte financeiro e estratégias eficazes para tornar disponível um tratamento adequado. ${ }^{15,17}$

Outro dado é que o evento de queda é considerado o mecanismo de lesão mais frequente entre idosos (cerca de 40\%), seguido pelos acidentes automobilísticos (28\% dos casos), além de atropelamentos $(10 \%)$ e até mesmo ferimento com arma branca e de fogo $(8 \%) .{ }^{17,18} \mathrm{Uma}$ pesquisa 
apontou que aproximadamente $88 \%$ dos idosos pesquisados que sofreram quedas têm medo de cair de novo, mostrando a possibilidade de o indivíduo restringir algumas tarefas por conta desta sensação e, por isso, ficarem suscetíveis a novas quedas. ${ }^{21}$

\section{Fatores associados}

A prevalência de quedas tem sido associadacom idade avançada, sedentarismo, autopercepção de saúde como ruim e maior consumo de medicações variadas de uso contínuo. Os estudos sugerem que tal procedimento seja repensado em casos de idosos com queixas desta enfermidade. Entre as quedas recorrentes, verificou-se a predominância maior em mulheres, idosos viúvos, solteiros e desquitados, idosos com história prévia de fratura, com algum grau de comprometimento nas atividades de vida diária e entre os que não referem leitura como atividade de lazer. ${ }^{18-22}$

Em pesquisa realizada com idosos institucionalizados, observou-se que o maior fator de risco ligado à queda é o número de doenças crônicas. Também se notou que incontinência urinária, polifarmácia, antidepressivos e arritmias atuam como situações de risco nesse ambiente. ${ }^{25}$

O sono adequado e o cochilo durante o dia parecem estar relacionados ao evento da queda. Um dos estudos mostrou que a insônia e o cochilo diurno são prováveis fatores. Estudo realizado com mais de 600 idosos mostrou uma prevalência de sintomas de insônia em quase metade dos participantes (49\%) que sofreram quedas. Entre os que relatavam cochilar durante o dia, a prevalência foi de $62 \%{ }^{19}$

No que diz respeito à ingestão de medicações, muitos deles fazem uso de vários fármacos, como benzodiazepínicos, neurolépticos e antidepressivos. A questão medicamentosa foi abordada por um trabalho que aponta que o uso de analgésicos narcóticos em pacientes com osteoartrite aumenta o risco de quedas. ${ }^{16}$ Outra pesquisa aponta que a prescrição de medicamentos cardiovasculares e psicotrópicos está associada com maior admissão em hospitais por quedas. Essa condição tem maior susceptibilidade em idoso de idades mais avançadas e com múltiplas comorbidades. ${ }^{14}$

O perfil de saúde dos idosos que caem inclui a hipertensão arterial sistêmica (HAS), insuficiência coronária, osteoporose e redução da acuidade visual gerada pela catarata. A diabetes mellitus apresenta consequências como decréscimo da função sensoriomotora, déficits neuromusculares e musculoesqueléticos e complicações farmacológicas. Em decorrência disso, a doença tem sido referenciada como de risco para quedas. ${ }^{14,18,23}$

O acometimento do segmento estrutural do corpo é um fator que apresenta importante relação com a queda, por reduzir a estabilização postural e das sobrecargas impostas. A osteoporose é considerada o grau avançado de perda da densidade óssea e deixa tais estruturas menos compactas e mais porosas. A fase da osteopenia, primeiro estágio deste processo, já sugere o desequilíbrio funcional entre osteoblastos e osteoclastos, células que trabalham na deposição da absorção óssea e que, quando comprometidas, aumentam o risco de a queda gerar fraturas. É importante lembrar que cada sexo tem sua peculiaridade quanto ao tempo e fatores de acometimento. A mulher, por exemplo, deve ficar mais atenta na menopausa, em que os níveis reduzidos de estrógeno contribuem para acelerar esse processo. . $^{1820,25}$

Um estudo prospectivo identificou que a fragilidade pode ser preditora da queda recorrente ou secundária. A inclusão de marcadores psicológicos e cognitivos na fragilidade está associada a quedas múltiplas. ${ }^{8}$ Fatores neurológicos que predispõem a quedas referemse àqueles que alteram a função vestibular, de propriocepção e cognição. Com isso, demências como Alzheimer, Parkinson ou desordens que dificultam a marcha e reduzem a acuidade visual estão inclusas. Estudos recentes corroboram trabalhos anteriores sobre a relação entre sintomas depressivos, identificados pela escala de depressão geriátrica, e quedas recorrentes. ${ }^{10,26}$ 
No que concerne ao ambiente doméstico, a Organização Mundial da Saúde (OMS) notificou que fatores como escadas irregulares, diferentes relevos de piso no mesmo ambiente, escadas com degraus mal projetados, ambientes externos ao redor da casa que, com a chuva (ou neve), apresentam o piso escorregadio, todos associados com baixa iluminação, são fatores que contribuem para a ocorrência de queda. ${ }^{12}$

Os ambientes que têm sido listados como locais de maior perigo no lar são, em primeiro lugar, o quarto, seguido por escadas e cozinha e, por último, sala de estar e banheiro. O quarto e sala de estar estão mais envolvidos com situações que inicialmente decorrem de tropeços sobre roupas no chão, sapatos, tapetes e demais objetos ou móveis. Os banheiros e cozinhas relacionam-se com pisos escorregadios, muitas vezes decorrentes da tarefa do idoso em fazer a limpeza destes. ${ }^{13,19}$

A escada, por exemplo, demonstra um alto risco quando se apresenta em design desfavorável, sem apoios, corrimãos ou placas antiderrapantes nos degraus. Esse cenário, somado à má iluminação local, com o idoso em dificuldade para locomover-se ou mesmo falta de equilíbrio, faz com este esteja mais vulnerável às quedas. No caso da escada, os estudos mostram que $75 \%$ das quedas nesse local ocorrem quando o idoso está descendo por ela, o que mostra a importância do autocontrole do corpo e da arquitetura favorável. ${ }^{13}$

O medo e a preocupação com novos acidentes domésticos pelos idosos também foram reportados e as atividades relatadas como as mais perigosas foram a limpeza da casa e o movimento de sentar e levantar. ${ }^{15,27}$

\section{Consequências da queda para o indivíduo idoso}

A importância do impacto da queda para a saúde pública resume-se a sua relação com altos níveis de morbidade e mortalidade, redução de funções, hospitalização, institucionalização e alto custo em saúde e serviços sociais. ${ }^{15,17}$
Segundo a percepção dos idosos e familiares, a dificuldade ou dependência para sentar e levantar, caminhar na superfície plana, tomar banho e caminhar fora de casa são algumas das consequências mais impactantes da queda. ${ }^{15,27}$ As diferenças entre gêneros também parecem influenciar na percepção da queda. O estudo de Gasparotto \& Santos apontou que, para os homens idosos, a queda passa a ser situação de risco quando ela interfere na sua capacidade de deslocar-se em ambientes externos ao lar. No caso das mulheres, estas se preocupam mais com as quedas quando a experiência de cair compromete a execução de atividades rotineiras do lar. É importante divulgar fatores como esses ao público idoso, de maneira a impactar na intenção e aderência do autocuidado. ${ }^{28}$

A queda recorrente pode dificultar mais ainda a atividade de caminhar. Estudos identificaram a redução de velocidade e comprimento da marcha em idosos que sofreram quedas e, comparandoos com indivíduos de mesma faixa etária e sem histórico de queda, verificou-se neste grupo uma dificuldade maior na tentativa de manter a marcha dentro dos parâmetros de normalidade. 1,21,25

No que diz respeito ao déficit de equilíbrio, os indivíduos que sofreram a queda apresentaram maior oscilação antero-posterior em posição ereta em relação aos que não caíram. Isso significa que é mais difícil manter o controle estático do corpo quando este sofre uma queda, o que pode contribuir, porém não isoladamente, para a recorrência destas. Este aparente desequilíbrio corporal foi maior em indivíduos acima dos 70 anos. Deve-se considerar também a tendência natural da perda do controle do corpo originada, entre outros, pela perda da força muscular ao longo dos anos. ${ }^{1,21,26}$

Um estudo avaliou os principais fatores associados às fraturas de quadril e consequente risco de queda. Quando a queda promove intensa dependência do idoso a ponto de restringi-lo ao leito, subsequentes quadros podem surgir. ${ }^{20} \mathrm{~A}$ perda funcional nas principais articulações do corpo (joelho, quadril, punhos), a diminuição da força muscular, a atrofia muscular por 
desuso, quadros de úlceras de decúbito na região sacral, trocantérica, calcânea e até mesmo occipital, deficiências respiratórias e problemas circulatórios são condições que, se não resolvidas, podem levar a óbito. ${ }^{18}$

O medo pode tornar-se um ciclo vicioso à medida que o indivíduo sofre a queda, fica apreensivo, para de movimentar-se com a desenvoltura anterior e assim reduz o equilíbrio e a mobilidade, predispondo-se a cair novamente. O medo de cair também está relacionado com pessoas que não sofreram nenhum episódio de queda. ${ }^{21,27}$

Há que se considerar o fato de que a queda em indivíduos idosos provoca também uma reação por parte dos familiares ou agregados que dividem a mesma moradia. É sabido que, após a queda, indivíduos que convivem com o idoso podem, por meio de seus atos aparentemente precavidos, facilitar o processo de dependência e o inevitável aumento da fragilidade. Com medo de que o evento ocorrido tenha episódios consecutivos, procura-se preventivamente restringir algumas atividades anteriormente executadas pelo idoso. ${ }^{6,10,27}$

Os dados mostram que a queda tem sido um fator adicional e agravante de grande influência entre as causas que limitam ou dificultam a execução das funções diárias dos idosos.

\section{Prevenção e intervenção nas quedas}

Prevenir quedas sempre será a opção mais eficaz e barata. Estudos mostram que as intervenções multifacetadas apresentam maiores chances de promover a conscientização na população de idosos. A atividade física atua como grande método de prevenção e pode ser adaptada aos déficits que já estejam presentes no idoso, como treino de marcha, de equilíbrio, cuidados com hipotensão postural e transferências., ${ }^{2,15,17}$

Segundo as Guidelines for the physiotherapy management of olderpeople at risk offalling, a prevenção deve englobar fatores multidimensionais para se atingir não somente um grau aceitável de adesão pelos pacientes, mas uma redução importante no número de quedas. Os autores indicam o trabalho de educação informativa sobre os fatores de risco e, em conjunto, trabalhos de força muscular, treino de equilíbrio e propriocepção. Atividades como tai-chi têm sido reportadas como importante alternativa de exercício de forte impacto na prevenção de quedas. O estudo ressalta a importância de se associar elementos diferenciados na abordagem preventiva. ${ }^{29}$

Ainda que o trabalho preventivo seja enfatizado como proposta determinante na redução das quedas, verificam-se algumas barreiras na implantação efetiva desse tipo de programa. É visto que idosos ainda têm dificuldade para reconhecer e conscientizar-se sobre os fatores de risco para quedas, e relatam muito pouco sobre essas questões aos médicos. Assim, muitas estratégias de prevenção acabam sendo negligenciadas, até que uma desordem física relevante, ocasionada por uma queda, possa ser um fator que estimule o idoso a se tratar. ${ }^{2,30}$

No que diz respeito aos cuidados hospitalares, o estudo de Cameron mostrou, após triagem de pesquisas recentes, que a reposição de vitamina D é importante coadjuvante na prevenção de quedas. O tratamento multifatorial é defendido em ambiente hospitalar, mas ainda segundo o estudo, os melhores resultados na prevenção de novas quedas dependem do nível da lesão e comprometimento funcional do paciente. ${ }^{31}$

Estudo de revisão sobre prevenção de quedas em idosos da comunidade aponta que a assistência multifatorial reduz as chances de quedas, porém ainda são pouco conclusivas em estudos de risco. O suporte dado no domicílio quanto à segurança do ambiente e aspectos ergonômicos, junto ao trabalho de exercícios físicos, é um exemplo de bons resultados na prevenção de quedas, principalmente quando se trata de idosos mais dependentes e com menor acuidade visual. ${ }^{3}$ Entre as intervenções individuais, o tai-chi permanece no topo dos exercícios de melhor impacto na prevenção de quedas. É necessário ressaltar que, apesar de referir-se a uma modalidade de treino, essa prática contribui em dois importantes aspectos (o muscular e o proprioceptivo), de 
maneira a promover benefícios mais amplos como os de equilíbrio e suporte muscular. ${ }^{30}$

Estudos recentes têm testado os efeitos da terapia manual como provedora de melhora no equilíbrio e estabilidade postural em pessoas de mais idade. ${ }^{32-34}$ Em revisão sistemática, um deles discute a tendência desta modalidade nos tratamentos de idosos, mas ainda com necessidade de mais estudos com metodologia avançada. ${ }^{32}$ Outro estudo aponta a necessidade de exercícios de fortalecimento da musculatura de tronco como proposta importante para equilíbrio funcional e redução de quedas. ${ }^{2}$

Corroborando outros estudos, uma metaanálise de dez anos também identificou que intervenções multifatoriais contribuíram para a redução de $14 \%$ sobre as quedas, porém exercícios específicos também mostraram afetividade. Intervenções sobre cuidados realizados em domićlio mostraram-se mais efetivas quando comparadas em atividades contemplando grupos da comunidade. ${ }^{30} \mathrm{O}$ estudo de Cabral et al. corrobora a ideia de intervenções multifacetadas trabalhando com treino de equilíbrio, exercícios supervisionados em domicilio, atividades educacionais e de modificação de comportamento. ${ }^{35}$

\section{REFERÊNCIAS}

1. Instituto Brasileiro de Geografia e Estatística. Indicadores Sociodemográficos e de Saúde no Brasil. Estudos \& Pesquisas: informação demográfica e socioeconômica, 25 [Internet] 2009 [acesso em 14 set 2011]:1-152. Disponível emhttp://www.ibge.gov.br/ home/estatistica/populacao/indic_sociosaude/2009/ indicsaude.pdf

2. Granacher U, Gollhofer A, Hortobágyi T, Kressig RW, Muehlbauer T. The importance of trunk muscle strength for balance, functional performance, and fall prevention in seniors: a systematic review. Sports Med 2013 Jul;43(7):627-41.

3. Gillespie LD, Robertson MC, Gillespie WJ, Sherrington C, Gates S, Clemson LM, et al. Interventions for preventing falls in older people living in the community. 2009 [alterado 2012 Set 12; acesso em 20 Set 2012]. In: The Cochrane Database
Ao ser considerado um estudo de atualização, os dados informados limitam-se apenas a uma parcela das informações divulgadas sobre a temática queda em idoso. É necessária constante atualização dos itens discorridos neste artigo e, para isso, sugere-se estudos de revisão sistemática como parâmetro de busca para maior amplitude de conhecimento.

\section{CONSIDERAÇÕES FINAIS}

É muito importante que profissionais de saúde que atuam diretamente com o público idoso conheçam o tema "quedas" nessa população. Uma vez que as intervenções e cuidados preventivos indicam associação de fatores ligados aos diversos aspectos, como controle medicamentoso, educação em saúde, exercícios e atividades físicas, acessibilidade, manutenção da capacidade funcional, entre outros, logo se conclui que, para atingir o ponto ideal de controle de quedas, é preciso que o idoso receba suporte multiprofissional.

Cabe então aos profissionais da área atentar para esse cenário, cada qual atuando em suas especificidades, e todos colaborando para a melhor condição de vida dos indivíduos idosos.

of Systematic Reviews [Internet]. Hoboken, NJ: John Willey \& Sons, Ltd. c2009 -. [cerca de 73 p.]. Disponível em: http://cochrane.bvsalud.org/doc.php $? \mathrm{db}=$ reviews\&id $=\mathrm{CD} 007146 \&$ lib $=$ COC Registro $\mathrm{n}^{\circ}$. CD007146.

4. Scliar, M. História do conceito de saúde. Physis: Rev Saúde Coletiva, Rio de Janeiro, 2007;17(1):29-31

5. D’Orsi E, Xavier AJ, Ramos LR. Trabalho, suporte social e lazer protegem os idosos da perda funcional: estudo epidoso. Rev Saúde Pública 2011;45(4): 685-92.

6. Maia FOM, Duarte YAO, Lebrão ML, Santos JLF. Risk factors for mortality among elderly peoples. Rev Saúde Pública 2006;40(6):2-7.

7. Murtagh KN, Hubert HB. Gender differences in physical disability among an elderly cohort. Am J Public Health 2004 Aug;94(8):1406-11. 
8. Vries JO, Peeters GMEE, Lips P, Deeg DJH. Does frailty predict increased risk of falls and fractures? A prospective population-based study. Osteoporos Int 2013;24(9):2397-403.

9. Coppin AK, Shumway-Cook A, Saczynski JS, Patel KV, Ble A, Ferrucci L, et al. Association of executive function and performance of dual-task physical tests among older adults: analyses from the InChianti study. Age ageing 2006 nov;35(6):619-24.

10. Ashburn A, Stack E, Ballinger C, Fazakarley L, Fitton C. The circumstances of falls among people with parkinson's disease and the use of falls diaries to facilitate reporting. Disabil Rehabil 2008;30(16):1205-12.

11. Damián J, Pastor-Barriuso R, Valderrama-Gama E, Pedro-Cuesta J. Factors associated with falls among older adults living in institutions. BMC Geriatr 2013;13(6):1-6.

12. World Health Organization. Global report on falls prevention in older age. France: WHO; 2007.

13. Siqueira FV, Facchini LA, Piccini RX, Tomasi E, Thumé E, Silveira DS, et al. Prevalência de quedas em idosos e fatores associados. Rev Saúde Pública 2007;41(5):749-56.

14. Payne RA, Abel GA, Simpson CR, Maxwell SR. Association between prescribing of cardiovascular and psychotropic medications and hospital admission for falls or fractures. Drugs Aging 2013 Apr;30(4):247-54.

15. Fabrício SCC, Rodrigues RAP, Costa Júnior ML. Causas e consequências de quedas de idosos atendidos em hospital público. Rev Saúde Pública 2004; 38(1):93-9.

16. Rolita L, Spegman A, Tang X, Cronstein BN. Greater number of narcotic analgesic prescriptions for osteoarthritis is associated with falls and fractures in elderly adults. J Am Geriatr Soc 2013 Mar;61(3):335-40.

17. Freitas MAV, Scheicher ME. Preocupação de idosos em relação a quedas. Rev Bras Geriatr Gerontol 2008;11(1):57-64.

18. Garcia R, Leme MD, Garcez-Leme LE. Evolution of brasilian elderly with hip fracture secondary to a fall. Clinics 2006 Dec;61(6):539-44.

19. Pereira AA, Ceolim MF, Neri AL. Association between insomnia symptoms, daytime napping, and falls in community-dwelling elderly. Cad Saúde Pública 2013 Mar;29(3):535-46.
20. Rossini M, Mattarei A, Braga V, Viapiana O, Zambarda $\mathrm{C}$, Benini C, et al. Risk factors for hip fracture in elderly persons. Reumatismo 2010;62(4):273-82.

21. Lopes KT, Costa DF, Santos LF, Castro DP, Bastone AC. Prevalência do medo de cair em uma população de idosos da comunidade e sua correlação com mobilidade, equilíbrio dinâmico, risco e histórico de quedas. Rev Bras Fisioter 2009;13(3):223-9.

22. Gama ZAS, Goméz-Conesa A. Factores de riesgo de caídas em ancianos: revisión sistemática. Rev Saúde Pública 2008;42(5):946-56

23. Crews RT, Yalla SV, Fleischer AE, Wu SC. A growing troubling triad: diabetes, aging, and falls. J Aging Res [Internet] 2013 [acesso em 18 Feb 2013]:1-17. Disponível em: http://www.ncbi.nlm.nih.gov/pmc/ articles/PMC3586503/

24. Ensrud KE, Ewing SK, Taylor BC, Fink HA, Stone KL, Cauley JA, et al. Frailty and risk of falls, fracture, and mortality in older women: the study of osteoporotic fractures. J Gerontol A Biol Sci Med Sci 2007;62(7):744-51.

25. Aikawa AC, Bracialli MP, Padula RS. Efeitos das alterações posturais e de equilíbrio estático nas quedas de idosos institucionalizados. Rev Ciênc Méd 2006;15(3):189-96.

26. Launay C, de Decker L, Annweiler C, Kabeshova A, Fantino B, Beauchet O. Association of depressive symptoms with recurrent falls: a cross-sectional elderly population based study and a systematic review. J Nutr Health Aging 2013 Feb;17(2):152-7.

27. Fabrício SCC, Rodrigues RAP. Percepção de idosos sobre alterações das atividades da vida diária após acidentes por queda. Rev Enferm UERJ 2006 Out/ Dez;14(4):531-7.

28. Gasparotto LPR, Santos JFFQ. A importância da análise dos gêneros para fisioterapeutas: enfoque nas quedas entre idosos. Fisioter Mov 2012 Out/ Dez;25(4):701-7.

29. Goodwin V, Briggs L. Guidelines for the Physiotherapy management of older people at risk of falling [Internet]. [S.1.]: AGILE falls guidelines working group; 2012 [acesso em 20 Set 2012]. 1-8 p. Disponível em: http://agile.csp.org. uk/news/2012/08/16/guidelines-physiotherapymanagement-older-people-risk-falling

30. Choi M, Hector M. Effectiveness of intervention programs in preventing falls: a systematic review of recent 10 years and meta-analysis. J Am Med Dir Assoc 2012 Feb;13(2):188.e13-21. 
31. Cameron ID, Gillespie LD, Robertson MC, Murray GR, Hill KD, Cumming RG, et al. Interventions for preventing falls in older people in care facilities and hospitals. 2009 [alterado 2012 Set 12; acesso em 20 Set 2012]. In: The Cochrane Database of Systematic Reviews [Internet]. Hoboken, NJ: John Willey \& Sons, Ltd. c2009 -. [cerca de 2 p.]. Disponível em: http://cochrane.bvsalud.org/doc.php?db=reviews \&id $=$ CD005465\&lib=COC Registro n ${ }^{\circ} .:$ CD005465

32. Holt KR, Haavik H, Elley CR. The effects of manual therapy on balance and falls: a systematic review. J Manip Physiol Ther 2012 Mar;35(3):227-34.

33. Howe TE, Rochester L, Neil F, Skelton DA, Ballinger C. Exercise for improving balance in older people. 2009 [alterado 2011; acesso em 14 set 2011]. In: The Cochrane Database of Systematic Reviews [Internet].
Hoboken, NJ: John Willey \& Sons, Ltd. c2009 -. [cerca de 2 p.]. Disponível em: http://cochrane. bvsalud.org/doc.php?db=reviews\&id=CD004963\&li $\mathrm{b}=$ COC Registro no.: CD004963

34. Sherrington C, Tiedemann A, Fairhall N, Close JCT, Lord SR. Exercise to prevent falls in older adults: an updated meta-analysis and best practice recommendations. NSW Public Health Bull 2011;22(4):78-83.

35. Cabral KN,Perracini MR, Soares AT, Stein FC, Sera CTN, Tiedemann A, et al. Effectiveness of a multifactorial falls prevention program in communitydwelling older people when compared to usual care: study protocol for a randomised controlled trial (Prevquedas Brazil). BMC Geriatr 2013 Mar;13:1-7. 